3. Theorem 1 of $\S 2$ concerned the consistence of the relations

$$
\begin{aligned}
& \phi\{x, \phi(y, z)\}=\phi\{y, \phi(x, z)\} \\
& \phi\{f(y, z), z\}=y \\
& f\{\phi(y, z), y\}=z .
\end{aligned}
$$

These relations, on the other hand, may be shown to be completely independent.* To do this, it suffices to exhibit eight systems, each of which satisfies or contradicts the preceding relations. Denoting by the symbol $(++-)$ that (6) is satisfied, $\left(6^{\prime}\right)$ is satisfied, $\left(6^{\prime \prime}\right)$ is contradicted, and so on, we have as the required systems:

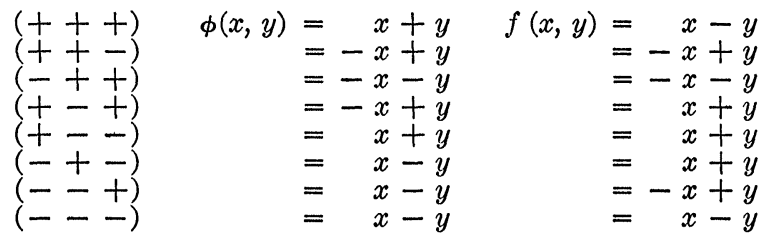

$$
\begin{aligned}
& \text { Chicago, IlL., } \\
& \text { July, } 1912 .
\end{aligned}
$$

\section{DOUBLE CURVES OF SURFACES PROJECTED FROM SPACE OF FOUR DIMENSIONS.}

BY DR. S. LEFSCHETZ.

(Read before the American Mathematical Society, September 10, 1912.)

1. IN the study of a surface $F$ in $S_{4}$, one of the problems is the determination of the genus $\pi^{\prime}$ of the double curve of a projection of $F$. Severit has shown that a general $F_{m}$ in $S_{4}$ has four fundamental projective characters, viz., the order $m$, the class $n$, the rank $a$ of a hyperplane section, and the number $t$ of trisecants through an arbitrary point 0 . He also gives an expression for the rank of the double curve in question, from which, knowing the number of pinch points $J$ of $F_{m}^{\prime}$ in projection of $F_{m}$, we can obtain $\pi^{\prime}$. It is not uninteresting p. 82,847 .

* Cf. E. H. Moore, The New Haven Mathematical Colloquium (1910), + "Intorno ai punti doppi impropri . . . ," Palermo Rendiconti, vol. 15 (1901), p. 32 . 
to show how $\pi^{\prime}$ can be obtained directly, and this is what we will do, discussing afterwards the case of a complete intersection in $S_{4}$.

2. If $h$ is the order of the double curve, we have

$$
\begin{gathered}
h=\frac{1}{2} m(n-1)-a, \\
p_{n}=\left(\begin{array}{c}
m-1 \\
3
\end{array}\right)-h(m-4)+\pi^{\prime}+2 t-1,
\end{gathered}
$$

where $p_{n}$ is the arithmetic genus of $F_{m}$. This gives

$$
\pi^{\prime}=h(m-4)+p_{n}-2 t-\left(\begin{array}{c}
m-1 \\
3
\end{array}\right)+1
$$

(1) $=\frac{1}{6}(n-1)\left[2 n^{2}-7 n-6\right]-\frac{1}{2} a(n-4)-2 t+p_{n}+1$.

Another method of obtaining $\pi^{\prime}$ could be used if we knew the genus $\pi$ of the curve of which the double curve is the projection. For the two are in $(2,1)$ relation, and the coincidences occur on the curve in $S_{4}$ only and are: (1) 2 for each triple point, or $2 t$; (2) one for each pinch point, or $j$, so that we have*

(2) $\therefore$

$$
2 t+j=2(\pi-1)+4\left(\pi^{\prime}-1\right),
$$

3 . If we wish to apply either method to the case of an $F_{\mu \nu}$, complete intersection of a $V_{\mu}$ and a $V_{\nu}$, we must calculate $t$, and for the first method we must find $p_{n}$. The other characters are indeed easily obtained.

$$
\begin{gathered}
m=\mu \nu, \\
h=\frac{1}{2} \mu \nu(\mu-1)(\nu-1), \\
\pi=\left(\begin{array}{l}
h \\
2
\end{array}\right)-2 h[2 h-\mu-\nu-(\mu-1)(\nu-1)+2], \\
j=\mu \nu(\mu-1)(\nu-1)=2 h .
\end{gathered}
$$

We obtain (5) by remarking that the curve in $S_{4}$ is the complete intersection of $V_{\mu}, V_{\nu}$ and a $V_{(\mu-1)(\nu-1)}$ and applying Veronese's $\dagger$ formula for the number of apparent double points of a curve in $n$-space, while (6) is obtained by remarking that $j$ is also the number of tangents drawn from $O$ to $F_{\mu \nu}$, and is therefore the number of points common to $V_{\mu}, V_{\nu}$ and their first polars with respect to $O$.

* Clebsch, Leçons de Géométrie, vol. II, p. 169.

† "Behandlung der projectivischen Verhältnisse . . . " Math. Ann., vol. 19. 
4. Value of $p_{n}$. It is easy to show that $F_{\mu \nu}$ is a regular surface. In fact the system $\left|G^{\prime}\right|$ adjoint to the system $|G|$ of the hyperplane sections of $F_{\mu \nu}$ is cut out by all the $V_{\mu+\nu-4}$ which do not pass through $F$, and they are cut by the hyperplane $H$ of $C$ in the system of all surfaces of order $\mu+\nu-4$ which do not contain $C$, surfaces which cut out on $C$ the complete canonic series on the curve.* $\left|C^{\prime}\right|$ cuts therefore on $C$ a series of defect $0 . \quad \therefore p_{g}-p_{n}=0, \dagger$ which was to be proved. To find $p_{g}$ we must obtain the number of conditions that a $V_{\mu+\nu-5}$ shall go through $F_{\mu \nu}$, since the canonic system of $F$ is cut out by the $V_{\mu+\nu-5}$ that do not go through $F_{\mu \nu}$. More generally we will propose to find the number $N_{l}$ of conditions that a $V_{l}$ shall go through $F_{\mu \nu}$. For this purpose we consider the curves $C_{i}$ obtained in cutting by hyperplanes $H_{i}$ $(i=1,2, \ldots, l), C_{i}$ is the complete intersection of two surfaces of order $\mu, \nu$ in $H_{i}$, and has for genus

$$
p=\frac{1}{2} \mu \nu(\mu+\nu-4)+1 .
$$

Let $r_{i}$ be the number of conditions that a $V_{l}$ going through $C_{1}, C_{2}, \ldots, C_{i-1}$ has to satisfy in order to go also through $C_{i}$. Such a variety already meets $C_{i}$ in $(i-1) \mu \nu$ points, contained in $i-1$ planes. The series $g_{(i-i+1) \mu \nu}^{r_{i}-1}$ which its variable intersections with $C_{i}$ cut on the curve is complete, since it is also cut out by a complete system of adjoints, namely the surfaces of order $l-i+1=\alpha$ of $H_{i}$, which go through the fixed group of $(i-1) \mu \nu$ points. But the surfaces of order $\alpha$ in $H_{i}$, cut out on $C_{i}$ a series of same degree, complete and contained in the preceding, therefore coincident with it. Hence if $\nu<\mu$ and

$$
\begin{aligned}
& \alpha<\nu, \text { then } r_{i}=\left(\begin{array}{c}
a+3 \\
3
\end{array}\right), \\
& \nu \leqq \alpha<\mu, \text { then } r_{i}=\left(\begin{array}{c}
\alpha+3 \\
3
\end{array}\right)-\left({ }^{\alpha-\nu+3}\right), \\
& \mu \leqq \alpha \leqq \mu+\nu-4, \text { then } \mu+\nu-4=\alpha \leqq \nu-4,
\end{aligned}
$$

and if $r_{i}-1$ is the residual special series, we have by applying Riemann-Roch's theorem

$$
\begin{gathered}
2\left(r_{i}-r_{i}^{\prime}\right)=2 \alpha \mu \nu-2(p-1) ; \\
\therefore r_{i}=\alpha \mu \nu-(p-1)+\left({ }^{\mu+\nu-a-1}\right),
\end{gathered}
$$

\footnotetext{
* Noether, "Zur Theorie des eindeutigen Entsprechens . . . ," Math Ann., vol. 8'(1874).

$\dagger$ E. Picard et Simart, Traité des fonctions algébriques de deux variables, 2 , pp. 437,489 .
} 


$$
\mu+\nu-4<\alpha .
$$

Then the series cut out by $V_{l}$ is not special.

It follows that

$$
\therefore r_{i}=\alpha \mu \nu-p+1 \text {. }
$$

$$
N_{l}=\sum_{1}^{l} r_{i}+1
$$

In particular

$$
N_{\mu+\nu-5}=\sum_{a=\mu-\nu}^{\mu-1}\left({ }_{3}^{+3}\right)+\sum_{a=\mu}^{\mu+\nu-5}(\alpha \mu \nu-p+1)+\sum_{a=1}^{\nu-4}\left({ }_{3}^{\nu-a}\right) .
$$

Also

$$
p_{g}=p_{n}=N_{\mu+\nu-5}
$$

hence $\left(1^{\circ}\right)$ If $l \geqq \mu+\nu-5 \geqq \mu \geqq \nu$,

$$
\begin{aligned}
N_{l} & =\sum_{a=\mu+\nu-4}^{l}(\alpha \mu \nu-p+1)+1+\left(p_{n}-1\right)+1 \\
& =\sum_{a=1}^{l}(\alpha \mu \nu-p+1)+p_{n}+1=\mu \nu\left({ }^{l+1}\right)-l(p-1)+p_{n}+1
\end{aligned}
$$

in virtue of the expression of $p$ * $^{*}$

$\left(2^{\circ}\right)$ If $l \leqq \mu+\nu-5$,

$$
N_{l}=p_{n}-\sum_{l+1}^{\mu+\nu-5}(\alpha \mu \nu-p+1)-\sum_{l-\mu+2}^{\nu-4}\left({ }^{\nu-\alpha}\right) .
$$

5. Value of $t$. Let $t=\varphi(\mu, \nu)$. Applying to a $V_{\nu}$ formed of a $V_{1}$ and a $V_{\nu-1}$, we have

$\varphi(\mu, \nu)=\varphi(\mu, \nu-1)+\mu\left(\begin{array}{l}\nu \\ 2\end{array}\right)(\nu-1)(\nu-2)-\mu(\mu-1)(\nu-1)(v-2)$,

where the second term is the number of bisecants of the surface $\left(V_{\mu}, V_{\nu-1}\right)$ meeting also the surface $\left(V_{\mu}, V_{1}\right)$, while the third is the number of these chords going through the intersection of $V_{\nu-1}, V_{\mu}$ and $V_{1}$. Taking $\sum_{1}^{5} \varphi(\mu, i)$ and simplifying, we obtain

$$
t=\varphi(\mu, \nu)=6\left(\begin{array}{c}
\mu \\
3
\end{array}\right)\left(\begin{array}{l}
\nu \\
3
\end{array}\right) .
$$

We see that (2) is easiest to apply in the case of a complete intersection, and indeed in this case comparing both values of $\pi^{\prime}$, we have the easiest way of obtaining $p_{n}$.

\footnotetext{
* Cf. Severi, "Su alcune questioni di postulazione," Palermo Rendiconti, vol. 17 (1903), p. 87.
} 
6. The curve of $S_{4}$ of which the double curve of $F_{\mu \nu}^{\prime}$ is the projection is a complete intersection, but such is not the case for the double curve itself. In this respect the case where $\mu=\nu=3$ is instructive. For then it is found that $t=6\left(\begin{array}{l}3 \\ 3\end{array}\right)=6, p_{g}=p_{n}=5$,

$$
\begin{aligned}
& \pi=5 \cdot 18+5-\left(\begin{array}{l}
8 \\
3
\end{array}\right)-2 \cdot 6+1=28, \\
& h=\frac{1}{2} 3 \cdot 3 \cdot 2 \cdot 2=18=6 \cdot 3=9 \cdot 2 .
\end{aligned}
$$

The complete intersection of an $F_{9}$ and an $F_{2}$ with six triple points is of genus 63, while the intersection of an $F_{6}$ and an $F_{3}$ with six triple points is of genus 28 , and yet the curve with the above characteristics cannot lie on an $F_{3}$, for then it would meet $F_{9}^{\prime}$ in a curve of order 36. If $F_{\nu(\mu-1)(\nu-1)}$ is the intersection of $V_{\nu}$ and $V_{(\mu-1)(\nu-1)}$, then the curve in $S_{4}$ is on $F_{\nu(\mu-1)(\nu-1)}$, the residual intersection being of order $\mu \nu(\mu-1)$ $(\nu-1)^{2}$, and its genus $p_{1}$ is found by remarking that if we project from another center this curve will be residual of one of order $\mu \nu(\mu-1)(\nu-1)$ and genus $\pi$, so that

$\pi_{1}{ }^{\prime}=\pi+\frac{1}{2} \mu \nu(\mu-1)(\nu-1)(\nu-2)[\mu \nu+\nu(\mu-1)(\nu-1)-4]$.

Thus, above, the residual curve is of order 72 and genus 397 .

LinColn, NeB.,

August 20, 1912.

\section{GEOMETRICAL OPTICS.}

The Principles and Methods of Geometrical Optics, especially as Applied to the Theory of Optical Instruments. By JAMES P. C. Southall. New York, The Macmillan Company, 1910. xxiii $+626 \mathrm{pp}$. with 170 figures.

Тнат mathematicians and physicists have left the field of geometrical optics so largely to the scientific experts of the best firms of optical engineers may be but one of the signs of our ever increasing specialization and its accompanying narrowing of interests. Yet the association of such names as Euler, Fermat, Gauss, Hamilton, Kummer, Moebius, Sturm shows that once mathematicians contributed largely to the subject and were inspired by it; a similar state of affairs is true in regard to physicists.

From an impartial viewpoint it can hardly be gainsaid that there are at present more points of contact between geometrical 